\title{
EVALUACIÓN DEL MANEJO DEL DOLOR Y FACTORES ASOCIADOS EN USUARIOS ADSCRITOS AL POLICLÍNICO DEL DOLOR Y CUIDADOS PALIATIVOS DEL HOSPITAL HERMINDA MARTÍN DE CHILLÁN, CHILE
}

\author{
Gloria Araneda Pagliotti
}

Licenciada en Enfermería. Magister Enf. Médico Quirúrgico

Héctor Acuña Ortiz*, Claudia Grez. Cádiz*, Marcelo Troncoso Véjar, Fernanda Zañartu Gaete*

* Licenciado/a en Enfermería

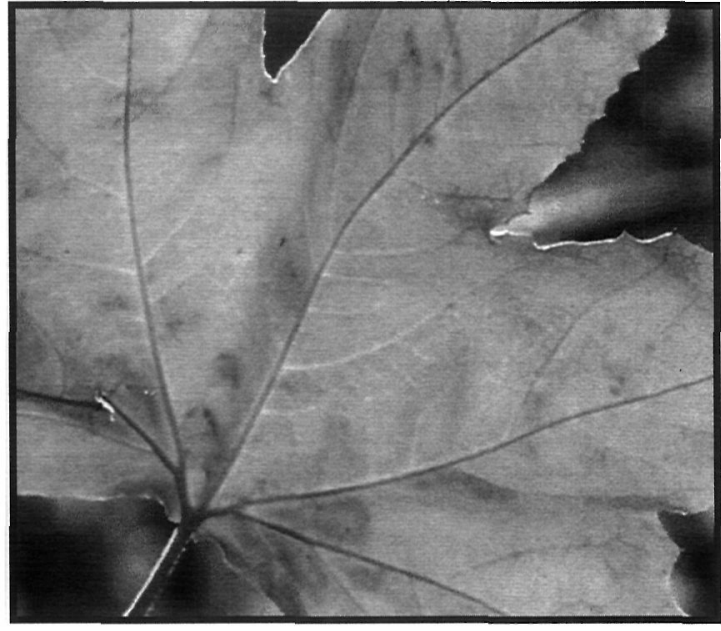

EVALUATION OF PAIN MANAGEMENT AND CONTRIBUTING FACTORS IN CLIENTS OF THE "PAIN RELIEF AND PALLIATIVE CARE POLYCLINIC", HERMINDA MARTIN HOSPITAL, CHILE

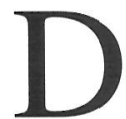
escriptive study whose aim was to identify pain management and evolution considering some contributing factors in adult and elder clients attending the Pain relief and palliative care Polyclinic at Herminda Martin Hospital, Chile.

A sample of 59 both male and female clients in easily accesible urban or rural areas were asked to answer a 29 item questionnaire during a home visit, whether they knew their diagnose or not. This questionnaire included the Scott-Huskinsson visual scale for pain and the WHO analgesic scale for classification of pharmacological treatment.
The results obtained showed that $64.4 \%$ of the sample did not complain of pain at the time, $49.2 \%$ is 66 years or older, $59.3 \%$ are female, $84.7 \%$ live in urban areas, $91.5 \%$ went to school, $88.1 \%$ are in benefit range $\mathrm{A}$ and $\mathrm{B}, 52.5 \%$ have a partner, the same percentage does not know their diagnose and 52.9\% have taken opioids for 1 to 4 months.

Statistically significant variables were the utilization and type of opioid for pain management.

Sociodemographic factors did not present significant statistical correlation.

Key words: pain, palliative care.

\section{RESUMEN}

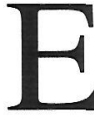
studio descriptivo, cuyo propósito fue identificar el manejo y evaluación del Dolor, considerando algunos de sus factores asociados en usuarios adultos y adultos mayores adscritos al Policlínico Alivio del Dolor y Cuidados paliativos del Hospital Herminda Martín de Chillán.

La muestra la constituyeron 59 usuarios, de ambos sexos, de zona urbana y rural, de fácil acceso, que se encontraran en su domicilio, que fueran capaces de contestar las preguntas realizadas, con y sin conocimiento del diagnóstico. Se les aplicó un cuestionario de 29 preguntas, elaborado por los autores, durante el mes de Abril, a través, de visita domiciliaria. Este incluyó la Escala Visual Análoga del Dolor (Scott - Huskinsson) y la Escala Analgésica de la OMS. para clasificación del tratamiento farmacológico. 
Los resultados obtenidos indican que el $64,4 \%$ de la muestra no presenta Dolor al momento de aplicar la encuesta, el 49,2\% tiene entre 66 y más años de edad, son de sexo femenino el $59,3 \%$, un $84,7 \%$ vive en el sector urbano, con escolaridad el $91,5 \%$, en tramo beneficiario A y B se encuentra el $88,1 \%$, con pareja el $52,5 \%$, este mismo porcentaje de usuarios no conoce su diagnóstico y el $52,9 \%$ utiliza opioides en un periodo de 1 a 4 meses.

Las variables que mostraron significancia estadística fueron el uso y tipo de opioide para el manejo del Dolor.

Se observó que los factores sociodemográficos no presentaron correlación estadística significativa.

Palabras clave: Dolor, cuidados paliativos

\section{INTRODUCCIÓN}

$\mathrm{E}$ 1 Cáncer es en la actualidad un grave problema mundial; afecta alrededor de 9 millones de casos nuevos al año y ocasiona la muerte a más de 5 millones de personas anualmente. Una de cada diez muertes en el mundo se deben a los diferentes tipos de Cáncer. En los países desarrollados esta relación es de 1 por cada 5 muertes. En América Latina se espera que para los próximos años, el Cáncer incremente en un 50\%. Entre $40 \%$ y $60 \%$ de los pacientes con diagnóstico de Cáncer, pueden presentar Dolor en las diferentes etapas de desarrollo de la enfermedad, éste aumenta a un $80 \%$ en la fase terminal.

El Dolor es el síntoma con mayor trascendencia para el paciente y su familia, es con seguridad el más temido y el que más interfiere en la calidad de vida del enfermo. Si bien éste, es el síntoma prioritario, es uno más en la constelación de síntomas que aquejan al paciente con Cáncer incurable, los no dolorosos se han denominado "síntomas secundarios o asociados al avanzado estado o curso de la enfermedad oncológica", éstos a su vez suelen sumarse a los derivados del tratamiento analgésico instaurado. Uno de los aspectos de mayor relevancia para intentar su alivio son los cuidados paliativos, tema que hoy en día ha sido poco estudiado, por ser una instancia en la medicina asistencial de desarrollo reciente, por lo cual el equipo de salud y especialmente, el profesional de enfermería tiene un rol muy importante en cuidados paliativos, pues está sometido a diario, al proceso continuo de la vida - enfermedad - muerte, y no como mero espectador, participa de la situación de los pacientes, ya que cuidar es su función primordial y además debe cumplir con las funciones asistenciales, educativas e investigativas.

La Enfermera es quien se encarga de realizar la valoración inicial del Dolor en relación a su semiología, debe poseer conocimientos amplios, actualizados y complementarlos con farmacología, estar alerta ante la presencia de efectos secundarios, buscar la forma de contrarrestarlos o de prevenirlos, debe educar a la familia en cuanto al cuidado y atención del paciente. Por último, siempre debe realizar un programa de evaluación, que le permita identificar el mantenimiento y/o mejoramiento de la calidad de vida del paciente oncológico terminal.

Esta investigación está dirigida a conocer el control del Dolor, la aparición de efectos molestos del tratamiento, derivados del esquema terapéutico que rige al Policlínico Alivio del Dolor y Cuidados Paliativos del Consultorio Adosado de Especialidades del Hospital Herminda Martín de Chillán.

Los cuidados paliativos "consisten en la atención activa, global e integral de las personas y sus familias que padecen una enfermedad, avanzada progresiva e incurable, con síntomas múltiples, intensos y cambiantes, que provocan gran impacto emocional en el enfermo, la familia o en el entorno afectivo y en el propio equipo, con pronóstico de vida limitado".

Los cuidados paliativos son un concepto de la atención al paciente y su familia que incluye: la asistencia total, activa y continuada por un equipo multiprofesional que proporcionan apoyo médico, psicológico y espiritual a los enfermos terminales y a sus seres queridos, cuando la expectativa médica no es la curación. La meta fundamental entonces, es dar calidad de vida mediante el control del Dolor y otros síntomas, para que el paciente pueda permanecer lo más alerta y cómodo posible. Debe cubrir entonces las necesidades físicas, psicológicas, espirituales y sociales del paciente y su familia.

Los cuidados paliativos proclaman el valor de la vida humana en cada momento e intentan aliviar al máximo el sufrimiento ocasionado por la enfermedad terminal. 
Es de importancia destacar que en 1967 la Enfermera Cicely Saunders fundó en Londres la primera Unidad de Cuidados Paliativos, dándose cuenta que si bien los enfermos terminales "podrían" tener un tratamiento para el Dolor de la enfermedad terminal, no tenían a su alcance el tratamiento de aspectos tan importantes como lo psicológico, espiritual y familiar.

Los instrumentos básicos en cuidados paliativos son:

1. Control de síntomas: evaluar y tratar adecuadamente los variados síntomas que puedan aparecer. Algunos se podrán controlar, como el Dolor y vómitos. Otros como la anorexia, enflaquecimiento, sólo implicará educar al paciente para su adaptación.

2. Apoyo emocional y comunicación: se debe realizar con el paciente, familia y equipo de salud.

3. Cambios en la organización: debe haber un trabajo en equipo y una adaptación a los cambios ocurridos en el paciente.

4. Equipo interdisciplinario: los cuidados paliativos deben ser planteados por un equipo que disponga de espacio, tiempo y actividades para ello.

Los términos Tumor, Neoplasia y Cáncer son a menudo utilizados como sinónimos para designar a las masas de tejido constituidas por células que proliferan con autonomía y de una forma desmesurada, sin obedecer a las leyes que controlan la multiplicación celular normal.

Neoplasia se refiere a la formación de un nuevo tejido, puede ser benigna o maligna según distintas características.

Epidemiológicamente, el Cáncer es la segunda causa de muerte en los países industrializados. Su incidencia y prevalencia es alta.

Alrededor de 5 millones de personas mueren anualmente en todo el mundo por Cáncer y se diagnostican cerca de 7 millones de casos nuevos. Según cifras de la OMS, la prevalencia por Cáncer en el mundo se acerca a los 14 millones de personas.

En Chile, el Cáncer constituye un importante problema de Salud Pública, es la segunda causa de muerte. En los últimos 30 años, han publicado la importancia relativa en ese período; extendiendo un leve predominio de las muertes del sexo masculino sobre el femenino. La región del país que mayor número de muertes acumula, es la región
Metropolitana, con 6.579, seguida de la Octava región con 2.230 y la Quinta región con 2.110. Se estima una incidencia de 247 casos de Cáncer por 100.000 habitantes. Con relación a la edad, el Cáncer es un problema del Adulto Mayor (63\% para el grupo de 65 años y más).

El Dolor ha sido definido por la Asociación Internacional para el Estudio del Dolor (IASP, Seattle, Washington) como: "Una desagradable experiencia sensorial y emocional que asocia a una lesión actual o potencial de los tejidos o que se describe en función de dicha lesión. El Dolor es siempre subjetivo. Cada individuo aprende a aplicar ese término a través de sus experiencias traumáticas juveniles. Indudablemente, se trata de una sensación en una o más partes del cuerpo, pero también es siempre desagradable y, por consiguiente, supone una experiencia emocional". Esta definición amplía aquella creencia del Dolor, como simplemente, un impulso nervioso desagradable, y la reemplaza por el concepto de experiencia dolorosa.

La evaluación del Dolor es una medida preliminar de gran importancia para el tratamiento satisfactorio del Dolor por Cáncer.

Exige un conocimiento del componente físico, como también de los psicológicos, espirituales, interpersonales, sociales y financieros, que integran el Dolor total del paciente.

Medir el Dolor es difícil, por su naturaleza subjetiva y por su carácter multidimensional. Es un fenómeno subjetivo que se trata de objetivar, con mucha variabilidad individual, y en que el mejor juez evaluador es el propio paciente.

Cada nuevo reporte del Dolor debe siempre llevar la evaluación inicial y debe identificar la causa del Dolor y desarrollar un plan de manejo. Las evaluaciones subsiguientes deben evaluar la eficacia del plan y en caso que el Dolor no se alivie, determinar si se debe a la progresión de la enfermedad, a una nueva causa de Dolor o al tratamiento oncológico.

A continuación se describen las principales fases de la evaluación del Dolor canceroso. La ignorancia de esas fases es una de las principales causas de los errores de diagnóstico y de los tratamientos inadecuados.

Tomar en serio la descripción que haga al paciente: Iniciar la conversación acerca del Dolor: 
para la evaluación inicial, el agente de salud deberá hacer al paciente preguntas específicas sobre el Dolor, en vez de basarse en una descripción espontánea. A veces, el paciente puede resistirse a admitir que tiene dolores ante el temor.

Evaluar la intensidad del Dolor: es preciso saber si el Dolor limita la actividad del paciente y altera su sueño, así como la medida en que la medicación o los métodos analgésicos producen alivio. Las escalas admitidas de grados de intensidad son útiles.

Tomar una historia detallada del Dolor: debe tomarse una historia detallada para determinar la localización y distribución del Dolor, sus características y su intensidad, su carácter continuo o intermitente y los factores que lo intensifican o lo alivian. Debe obtenerse asimismo información sobre cualquier tipo de debilidad y disfunción sensorial.

Evaluar el estado psicológico del paciente: la información sobre enfermedades anteriores, grado actual de ansiedad y depresión, ideas suicidas y grado de discapacidad funcional facilitan la detección de pacientes que pueden requerir un apoyo psicológico más específico.

Efectuar un examen físico minucioso: para determinar la causa del Dolor e iniciar el tratamiento apropiado a veces basta con una historia minuciosa y un examen clínico detenido.

Considerar otros métodos del control del Dolor: aunque los fármacos son el elemento principal en el tratamiento de los dolores por Cáncer, la consideración de otros métodos posibles es muy ventajosa en algunos casos.

Observación de los resultados del tratamiento: la evaluación y el tratamiento continuos exigirán un trabajo en equipo y dependerán en gran medida de las observaciones del agente de salud que dispensa la asistencia.

Entre las numerosas barreras que existen para una adecuada evaluación del Dolor, tenemos:

1. La naturaleza multidimensional de la experiencia subjetiva del Dolor.

2. La falta de un lenguaje claro para expresar el Dolor.

3. La poca comunicación entre el paciente y el médico acerca del Dolor.

$\mathrm{Al}$ evaluar el Dolor considerar:
- Intensidad: ¿Cuánto?

- Cualidad: ¿Cómo?

- Localización: ¿Dónde?

- Conducta dolorosa: ¿Qué hace para disminuir su Dolor?

- Evaluación psicológica.

Esto hace que no siempre sea fácil la valoración del Dolor mediante un solo método.

Existen distintos métodos para medir el Dolor:

\section{Métodos Objetivos:}

Observación directa de la conducta dolorosa, tiene relación con la actividad diaria, como: patrón de sueño, alimentación, actividad laboral, recreación, signos de dolor como gemidos, alteraciones en el ánimo, relaciones personales, etc. Estos métodos no cuantifican directamente el Dolor.

\section{Métodos Subjetivos:}

Se basan en el autorreporte del Dolor por el paciente. Los instrumentos utilizados con mayor frecuencia son las escalas de intensidad del Dolor, que miden sólo el aspecto intensidad.

La escala Visual Análoga (E.V.A. Scott Huskinsson): permite al paciente expresar la gravedad de su Dolor, transformándolo en un valor numérico. Consiste en una línea recta, habitualmente de $10 \mathrm{~cm}$. de longitud, con las leyendas "SIN DOLOR" y "DOLOR MÁXIMO" en cada extremo. El paciente anota en la línea el grado de Dolor que siente de acuerdo a su percepción individual, midiendo el Dolor en centímetros desde el punto cero (SIN DOLOR). También puede confrontarse con escalas semejantes que en un extremo tengan "SIN ABOLICIÓN DEL DOLOR" y en el otro "AUSENCIA DE DOLOR" o "MÁXIMA ABOLICIÓN". La EVA es hoy de uso universal. Es un método relativamente simple, que ocupa poco tiempo, aún cuando requiere de un cierto grado de comprensión y de colaboración por parte del paciente.

Esta escala tiene varias ventajas entre las cuales se encuentran: buena sensibilidad, simplicidad, uniformidad y confiabilidad para medir la intensidad del Dolor.

La escalera analgésica de la OMS es un método simple, eficiente y de bajo costo que asegura 
una prescripción racional para el paciente individual. Esta recomendación surgió de un Comité de expertos convocado por la OMS y publicado por primera vez en 1986. Posteriormente este método ha sido revisado y perfeccionado en sucesivas oportunidades (1990 y 1994).

Se ha comprobado que la aplicación de esta aproximación terapéutica consigue aliviar el Dolor en el $90 \%$ de los pacientes y sobre el $75 \%$ de los pacientes terminales.

Los principios fundamentales de la escalera analgésicas son:

La escalera analgésica de la OMS es un método simple, eficiente y de bajo costo que asegura una prescripción racional para el paciente individual. Esta recomendación surgió de un Comité de expertos convocado por la OMS y publicado por primera vez en 1986. Posteriormente este método ha sido revisado y perfeccionado en sucesivas oportunidades (1990 y 1994).

Se ha comprobado que la aplicación de esta aproximación terapéutica consigue aliviar el Dolor en el $90 \%$ de los pacientes y sobre el $75 \%$ de los pacientes terminales.

Los principios fundamentales de la escalera analgésicas son:

- Vía oral.

- Reloj en mano.

- El principio en Escalera.

- La modalidad determinada según el sujeto.

Por lo anterior, es que a través de un estudio descriptivo se propuso identificar el manejo y evaluación del Dolor, valorando algunos de los factores relacionados que se presentan en su control, en los usuarios adultos y adultos mayores del Policlínico Alivio del Dolor y Cuidados Paliativos del Consultorio Adosado de Especialidades del Hospital Herminda Martín de Chillán, con la finalidad de diferenciar la influencia de las características sociodemográficas como también las distintas alternativas de tratamiento utilizado en los pacientes con Cáncer terminal.

\section{MATERIAL Y MÉTODOS}

Estudio Descriptivo de corte Transversal que consideró a 117 usuarios adscritos al Policlínico Alivio del Dolor y Cuidados Paliativos del Consultorio Adosado de Especialidades del
Hospital Herminda Martín de Chillán. Sin embargo, por las características de los pacientes el estudio a aquellos que cumplan con las siguientes condiciones: Usuarios adultos y adultos mayores, de ambos sexos, de zona urbana y rural, de fácil acceso, que se encuentren en su domicilio, que sean capaces de contestar a las preguntas realizadas, con y sin conocimiento del diagnóstico, adscritos al Policlínico; por lo tanto el universo corresponde a 59 pacientes.

Escala Visual Análoga (EVA) (Scott Huskinsson):

Escala para medir el Dolor, aprobada por la OMS, consiste en una línea recta, de 10 centímetros de longitud, con las leyendas "sin Dolor" y "Dolor máximo" en cada extremo. Permite al paciente expresar la gravedad de su Dolor, transformándolo en un valor numérico.
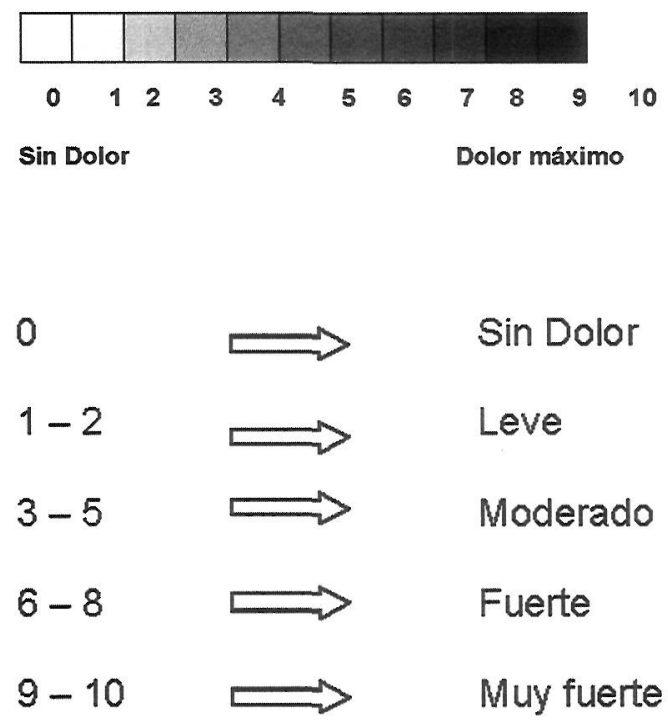

\section{Escala Analgésica de la OMS:}

Esta escala muestra el uso secuencial de los medicamentos. El primer peldaño consistirá en usar un medicamento no opioide. Si éste no alivia el Dolor se debe añadir un opioide contra el Dolor de leve a moderado. Si esa combinación no surte efecto, habrá que utilizar un opioide para el Dolor de moderado a agudo. No hay que usar al mismo tiempo más de un medicamento de cada grupo. Cuando esté específicamente indicado, se adminis- 
trarán medicamentos coadyuvantes. Para el manejo del Dolor muy intenso, se aplicaran medidas analgésicas invasivas.

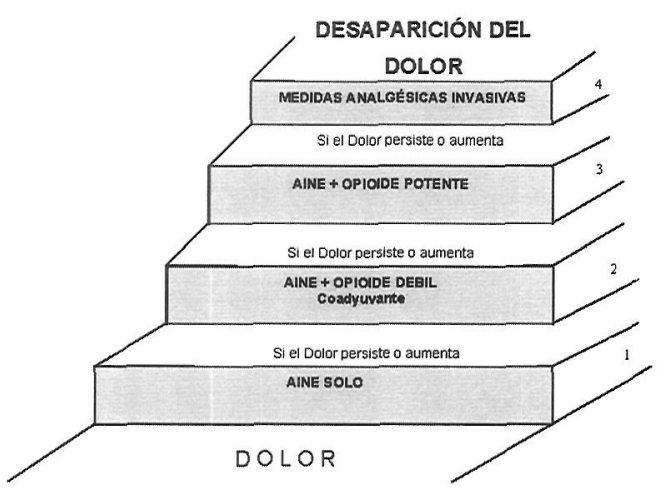

El cuestionario aplicado a los adultos y adultos mayores será codificado y procesado a través de sistema computacional (Epi Info 2000), y prueba estadística gamma.

\section{ANÁLISIS DE DATOS}

\section{GRÁFICO Nº 1 : DOLOR EN VISITA DOMI- CILIARIA}

Los resultados obtenidos señalaron que $64,4 \%$ de los usuarios no presentaron Dolor y el 35,6\% refería Dolor.

\section{GRÁFICO Nº 2: DOLOR SEGÚN EVA}

Considerando el porcentaje de usuarios que manifestaron Dolor, el 8,5\% tiene Dolor leve, el $16,9 \%$ moderado, $10,3 \%$ fuerte y $0 \%$ de la población estudiada no presentó Dolor muy fuerte, lo que demuestra un efectivo manejo del Dolor.

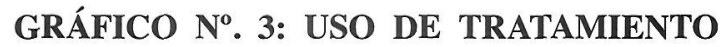 ALTERNATIVO}

En relación al gráfico $\mathrm{N}^{\circ} 3$ se observaron los siguientes resultados, el $83,1 \%$ de la población utilizaba un tipo de tratamiento alternativo, aparte del tratamiento farmacológico indicado en el consultorio, que consistió mayoritariamente en medicina de herbolario y masaje, mientras que un $16,9 \%$ de los usuarios encuestados no utilizaba tratamiento alternativo.

\section{RELACIÓN ENTRE USO DE OPIOIDE Y DOLOR SEGÚN EVA}

La tabla $\mathrm{N}^{\circ} 14$ indica que dentro del grupo de pacientes que no usan opioides $(13,6 \%)$ hay buen manejo del Dolor, un solo usuario refiere Dolor fuerte en esta categoría.

Un $86,4 \%$ de los pacientes utilizan opioides para el manejo del Dolor, de los cuales el $60,8 \%$ no refieren Dolor, apreciándose significancia estadística entre las variables.

\section{RELACIÓN ENTRE TIPO DE OPIOIDE Y DOLOR SEGÚN EVA}

Los resultados obtenidos son estadísticamente significativos, observándose que de los 51 usuarios que utilizan opioides, los más usados son codeína y morfina, que a su vez representan el más alto porcentaje de pacientes con ausencia del Dolor (75\% y $43,8 \%$ respectivamente). Mientras que con tramadol y oxicodona, hay más altos porcentajes de presencia de Dolor en sus distintas intensidades.

\section{CONCLUSIONES}

Los resultados obtenidos señalan que:

- Se puede concluir que existe un buen manejo del Dolor, ya que no se encontraron pacientes con Dolor muy fuerte.

- Los factores sociodemográficos no tienen significancia estadística al relacionarlos con el Dolor, sin embargo, la mayoría de los usuarios pertenece a la edad 51 años y más, sólo en ellos se presenta Dolor fuerte.

- Se observó una mayor cantidad de usuarios de sexo femenino los cuales presentaron Dolor fuerte.

- En los pacientes casados o con pareja se observó menor porcentaje de Dolor fuerte en comparación con los solteros, viudos o separados.

- Los dos factores que resultaron significativos con el manejo del Dolor fueron:

- El uso de opioide; está directa y proporcionalmente relacionado con el manejo del Dolor.

- El tipo de opioide; codeína y morfina son los opioides más utilizados y en ambos se presentaron los más altos porcentajes de pacientes sin Dolor.

- Se cumplen satisfactoriamente los principios de la escala analgésica de la OMS, ya que los pacientes con AINES sólo, se encuentran en su mayoría sin Dolor, al igual que los usuarios que 
utilizan opioide también presentan porcentajes altos de ausencia de Dolor.

- Debido al prolongado tiempo con uso de opioide, aparecen mayores casos de Dolor moderado y fuerte, haciendo necesaria la valoración constante.

\section{GRÁFICO No 1: DOLOR EN VISITA DOMI- CILIARIA}

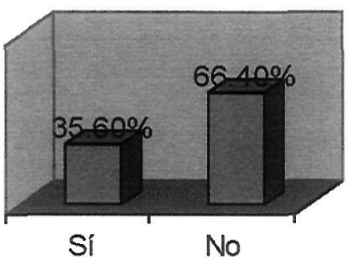

Serie1

Sí No

$\mathrm{n}: 59$

FUENTE: EVALUACIÓN DEL MANEJO DEL DOLOR Y FACTORES ASOCIADOS EN USUARIOS ADSCRITOS AL POLICLÍNICO ALIVIO DEL DOLOR Y CUIDADOS PALIATIVOS DEL HOSPITAL HERMINDA MARTÍN DE CHILLÁN.

\section{GRÁFICO No . 2: DOLOR SEGÚN EVA}

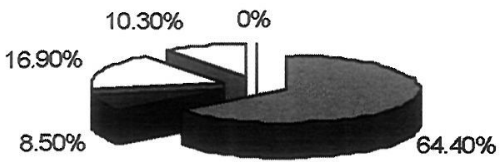

$\square$ Sin Dolor $\square$ Leve $\square$ Moderado $\square$ Fuerte $\square$ Muy Fuerte FUENTE: IDEM GRAFICO No $1 \quad$ n: 59

\section{GRÁFICO No. 3: USO DE TRATAMIENTO ALTERNATIVO}

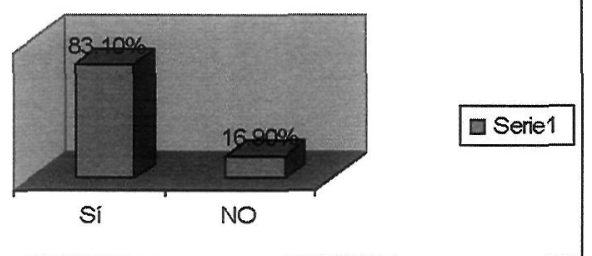

\section{RELACIÓN ENTRE USO DE OPIOIDE Y DOLOR SEGÚN EVA}

\begin{tabular}{|c|l|l|l|l|}
\hline \multirow{2}{*}{$\begin{array}{c}\text { Dolor según } \\
\text { EVA }\end{array}$} & \multicolumn{2}{c|}{ No } & \multicolumn{2}{c|}{ Si } \\
\cline { 2 - 5 }$(0)$ & 7 & 87,5 & 31 & 60,8 \\
\hline $\begin{array}{c}\text { Sin Dolor } \\
(1-2)\end{array}$ & 0 & 0 & 5 & 9,8 \\
\hline $\begin{array}{c}\text { Leve } \\
\text { Moderado }\end{array}$ & 0 & 0 & 10 & 19,6 \\
\hline $\begin{array}{c}\text { Fuerte } \\
(6-8)\end{array}$ & 1 & 12,5 & 5 & 9,8 \\
\hline $\begin{array}{c}\text { Total } \\
\text { FUENTE: IDEM GRÁFICON }\end{array}$ & 8 & 13,6 & 51 & 86,4 \\
\hline
\end{tabular}

\section{RELACIÓN ENTRE TIPO DE OPIOIDE Y DOLOR SEGÚN EVA}

\begin{tabular}{|c|c|c|c|c|c|c|c|c|}
\hline Dolor según & & de. & & orfi. & & ama. & & sico. \\
\hline EVA & $\mathbf{N}^{\circ}$ & $\%$ & $\mathbf{N}$ & $\%$ & $\mathbf{N}^{\circ}$ & $\%$ & $\mathbf{N}^{\circ}$ & $\%$ \\
\hline $\begin{array}{c}\text { Sin Dolor } \\
(0)\end{array}$ & 24 & 75 & 7 & 43,8 & 0 & 0 & 0 & 0 \\
\hline $\begin{array}{l}\text { Leve } \\
\qquad(1-2)\end{array}$ & 2 & 6,3 & 2 & 12,5 & 1 & 50 & 0 & 0 \\
\hline $\begin{array}{l}\text { Moderado } \\
\qquad(3-5)\end{array}$ & 4 & 12,5 & 5 & 31,3 & 0 & 0 & 1 & 100 \\
\hline $\begin{array}{l}\text { Fuerte } \\
(6-8)\end{array}$ & 2 & 6,3 & 2 & 12,5 & 1 & 50 & 0 & 0 \\
\hline Total & 32 & 62,7 & 16 & 21,4 & 2 & 3,9 & 1 & 2 \\
\hline
\end{tabular}

\section{BIBLIOGRAFÍA}

- Acello, B. (2000). Cumplimiento de las normas de acreditación para el Control del Dolor. Nursing volumen 18,8 , pp. 13-15.

- Araneda, G. et al. (2001) El concepto de cuidar en Enfermería. Fondo Desarrollo y Docencia. Universidad del Bío - Bío, Chile p. 6-9.

- Arriagada, A. y Schurmann, S. (1997) Instrumentos Básicos en cuidados Paliativos. Universidad La Frontera, Temuco, pp. 55-58.

- Arriagada, A. y Schurmann, S. (1997) Acerca del Dolor y Cuidados Paliativos. Temuco Universidad La Frontera,. pp. 61-71.

- Bilbeny, L. (1997) El Dolor, Aspectos Básicos y Clínicos. Mediterráneo,pp. 145-161. 
- Bilbeny, N. et al. (1997) El Dolor Aspectos Básicos y Clínicos. Mediterráneo,. pp. 385-399.

- Castro, M. y Col. (1995). Dolor Crónico, En el Dolor. 25. pp. 9-16.

- Cortés, C. y Col. (2001). Alivio del Dolor y Cuidados Paliativos, el enfrentamiento de los enfermos de Cáncer al diagnóstico terminal. Chillán, Chile. Universidad del Bío Bío. 125 p. (Tesis grado Licenciatura, Enfermería).

- Cruces, L. Cuidados Paliativos. (fecha de consulta 2001). En

Internethttp://www.paliativos.com/dolor3.html.

- Cruces, L. (2000) El Dolor. 32, 23-29.

- Cuenca, JL et al.(1998). El Dolor, 24,16-18.

- Daudt, A. et al. (1998).Opioides en el manejo del Dolor, uso correcto o subestimado? hospital universitario. Assoc. médica Brasil.

- Departamento de Salud. Los Cuidados paliativos. (fecha consulta 2001).En Internet http://www.cfnavarra.es/Salud/CONSEJOS/paliativos.htm

- Derderian, T.(1998) Historia del Tratamiento del Dolor 26, 6-9.

- Derio, M.L. et al (1998). "Programa Nacional Alivio del Dolor y Cuidados Paliativos" serie 01 Chile Ministerio de Salud. pp. 9-16. (Norma de Enfermería)

- Encarta Enciclopedia. Medicina Alternativa. (CD Rom ). Microsoft Corporation 2000. Disco Compacto.

- Farreras, R. Manual de Medicina Interna. [CD Rom]. Edición 13, 1 disco compacto.

- Gador S.A. Cuidados Paliativos en Internet http://www.gador.com.ar/iyd/onco/cpaliativos.htm.

- Ganong, F. (1997) Manual de Fisiología Médica. Manual Moderno. pp. 89-100.

- Medición del Dolor en Clínica,( fecha consulta 2001). En Internet http: // escuela.med.puc.cl/paginas/publicaciones/Boletin/htlm/ dolor/3_4.htlm.

- García, H. A. (1996) Formación en Cuidados Paliativos. España. En Internet //members.es.tripod.de/sociedad/forma.htm.

- Huñis, A et al. (1998).Dolor por Cáncer, Buenos Aires. 29 p.

- Instituto Europeo de Medicina Virtual. Enfermo Crónico. (fecha de consulta 2001). En Internet http://www.worldwidehospital.com/h24h/palbas.htm.

- Jacox, A y Col. (1994) Manejo del Dolor por Cáncer. U.S. Department of Health and Human Services.pp. 23-38.

- Jara, V . (1999) El Dolor. 31,9-15.

- Kettelman, K. (2000) ¿Por qué administrar más morfina a un paciente moribundo?. Nursing. volumen 18, 4,28-29.

- Keegan, L. (1998). Nursing. volumen 16, 9, 6-9.
- López, E.(1998) Enfermería en Cuidados Paliativos. Panamericana Madrid.

- Mc Ceffery, M. et. al. (1999) . Nursing. volumen 17, 7,8-11.

- National Cáncer Institute. (fecha consulta 2001). Cuidados Paliativos. En Internet http://cancernet.nci.nih.gov/clinpdq/factsspan2/600086.html.

- Oncología y Cuidados Paliativos (fecha de consulta 2001). En http://www.pharma.es.novartis.com/preguntas/onco00.html.

- Organización Mundial de la Salud. (1996) Alivio del Dolor en el Cáncer. Con una guía sobre la disponibilidad de opioide. Ginebra, pp.3-5.

- Pacile Carlos et al, (1997) El Dolor. Aspectos Básicos y Clínicos. Mediterráneo, pp.248-257.

- Programa de Cuidados Paliativos. (fecha consulta 2001). En Internet http://www.paliativos.com/program.html.

- PR Vademecum. Chile: EDITORA Ltda. 2000. p. LXIII,CI, CLXI, CLXXVII, CCXXXI-CCXXXII.

- Pereira, J. et. al. (1997) Manual de Cuidados Paliativos de Edmonton. Universidad de Alberta .Canadá. pp.10-14.

- Redondo, B. et al. Analgesia morfínica intraventricular en el Dolor por Cáncer, (fecha de consulta 2001) En Internet

http://bvs.sld.cu/revistas/onc/vol12_1_96/onc03196.htm

- Salgado, C. et al. (1998) Evaluación del dolor en pacientes oncológicos que acuden a los servicios de urgencias. Assoc. Médica. (Base de Datos Lilacs).

- Sepúlveda, M, Et al. (1999) Programa Nacional Alivio del Dolor y Cuidados Paliativos a Pacientes con Cáncer. serie 01, Chile, Ministerio de Salud. p. 19-39. (Normas).

- Silva, M. (1995) Dolor Crónico y Dolor por Cáncer. Laboratorio Andrómaco S.A. Chile. p p.25-33.

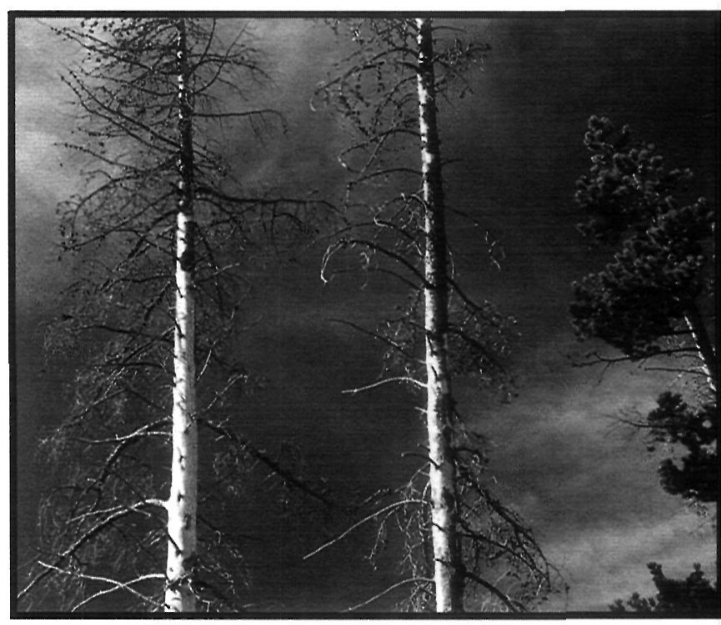

